\title{
TERCER SECTOR E INSERCIÓN SOCIAL
}

\section{JOAQUÍN GARCÍA ROCA}

Profesor de la E.U. de Trabajo Social. Departamento de Trabajo Social y Servicios Sociales. Universidad de Valencia.

\section{INTRODUCCIÓN}

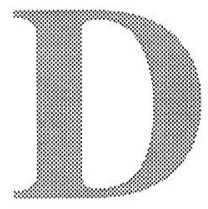

espués de un tiempo en el que el escenario de la margina-lidad estaba ocupado por ciertas figuras tradicionales (mendigos y vagabundos, transeúntes e indigentes...) hemos asistido en las últimas décadas a un cambio de personajes y también de decorado. En la nueva escena, los personajes tradicionales asumen papeles secundarios, y se produce un cambio profundo en el reparto: han aparecido en escena los parados de larga duración, los jóvenes sin empleo, las personas golpeadas por la drogodependencia, las familias monoparentales, las mujeres con responsabilidades familiares, la violencia doméstica, la inadaptación juvenil, la inmigración económica..., todos ellos crecen en las fronteras de un nicho de bienestar y privilegio como islas habitadas por todos aquéllos que no han podido pagar el precio de la integración social: han quedado fuera del trabajo regular, de la vivienda decente, de la cohesión social, de la convivencia estable y de las instituciones de socializacion. Permanecen fuera de las grandes corrientes por donde circulan los dinamismos de la sociedad, se reproducen entre ellos, viven de prestaciones y pasan de todos los esfuerzos bien intencionados de quienes quieren moralizarles y normalizarles; los excluidos, jóvenes o adultos, viven su humillación aislándose o buscando refugio en el alcohol, la droga y la autodestrucción.

¿Qué tienen de común todas estas figuras y estos espacios que han ido cristalizando en una intensa geografía social que va desde ciertos subgrupos que habitan en barrios desheredados hasta los alumnos de escolaridad fracasada con deficiencias múltiples, inadaptados sociales, familias mal socializadas y personas "inempleables"?

\section{EL NUEVO ESTATUTO DE LA EXCLUSIÓN.}

En primer lugar, comparten el estatuto de la complejidad que rompe cualquier aproximación sustentada en causalidades simples e invalida 
cualquier aproximación en sede a problema-causa-remedio. En su lugar, se domicilian en la interrelación de todos sus componentes: sin vivienda no hay trabajo, sin trabajo no hay vivienda y sin trabajo ni vivienda no hay participación. Se realiza como multiproblema ya que a las dificultades económicas se le añaden las sociales, las étnicas y las culturales. La desorientación, que sentimos a pie de obra, es la interrelación de muchas desventajas juntas que se retroalimentan mutuamente: la renta, el paro, la baja cualificación, la vivienda inadecuada, la mala salud, la ruptura familiar, la insignificancia y la desafiliación.

En segundo lugar, la exclusión no está donde están los excluidos, sino que anda por otra parte, entre tramas y marañas; nos permite constatar la existencia de una población sobrante que no está donde quisiera estar; «nadie puede, a priori, ser considerado responsable de su exclusión; es un sufrimiento y una injusticia tal que ninguna de sus víctimas podría desearla para sí»' Debe, en consecuencia, distanciarse de cualquier consideración que la confunda con la culpabilidad de quien la sufre. Hay que afirmar inequívocamente esta dimensión porque hay muchos empeñados en culpabilizar la exclusión y convertirla en un acto de voluntad; con frecuencia se escucha que son personas que se lo merecen, que se lo han ganado, que están ahí porque quieren. Resultan radicalmente falsos los intentos de identificar exclusión y culpabilidad, como si cada uno estuviera allí donde ha elegido estar. No se les puede culpabilizar por la situación de no-trabajo que ellos no han elegido, como quiere el actual clima neoliberal. La paradoja de la inserción consiste en ocuparse de validos invalidados por la coyuntura. De este modo la exclusión se emparenta con la víctima y se hermana con la injusticia. Cuando se olvida este aspecto, se impone una aproximación administrativa a la exclusión que la convierte en un fenómeno totalmente natural, de este modo quedan acreditados para controlarla, manipularla y privarle del carácter de violencia. Queda de este modo a disposición de medidas legales y policiales. Son pocos los que relacionan el paro, la pobreza, la marginalización o la exclusión con la injusticia. Lo que es grave. «El sufrimiento sufrido por los excluidos es una desdicha que no provoca necesariamente una reacción política; a lo sumo puede suscitar compasión, piedad, caridad; ni siquiera convoca necesariamente a la indignación, a la cólera o a la protesta colectiva. El sufrimiento sólo suscita movimientos de solidaridad y de protesta cuando se asocie el sufrimiento del otro con la convicción de que este sufrimiento es injusto.» ${ }^{2}$

CEE. Dictamen sobre la pobreza del Comité Económico y Social, sesión 268 del 12 de junio 1989. en Diario oficial de las Comunidades Europeas, 28 agosto 1989.

2 DEJOURS, $\mathrm{CH}$. Souffrance en France. La banalisation de l'injustice sociale. Seuil, París, 1998, p.17-18. 
En tercer lugar, al remitir el problema de la exclusión fuera de sí mismo, no se quiere convertir en una variable del sistema, o un capítulo del destino social, como se pretende desde algunas cátedras e incluso púlpitos, sino que el mundo de la exclusión incorpora al sujeto con una extraña complicidad entre estructura y acción. La exclusión es, en la actual realización de la marginalidad, el punto final de unas posibilidades humanas, la expropiación del ser humano y la interrupción de sus relaciones interhumanas. Estamos ante un proceso que pone en cuestión al sujeto, pero no lo elimina. Es necesario pasar del trayecto al proyecto y afirmar que son ellos también los responsables de su propio fracaso o de su propio éxito, son ellos mismos los protagonistas de su propio aprendizaje. ${ }^{3}$ Interesa subrayar este aspecto para afrontar la actual geopolítica de la impotencia que se basa en anular las capacidades de las personas y sus acciones colectivas en función de unos poderes superiores que les convierten en meros satélites.

En la actualidad somos reos de la ilusión económica que ha invadido todos los rincones de la vida, y ha sometido la forma de amar y de desear, de esperar y desesperar a la dictadura del mercado. Esta prepotencia de lo económico genera la idea de que las víctimas no pueden hacer otra cosa que mostrar las contradicciones del sistema. $\mathrm{Ni}$ unos ni otros creen en la formación de actores sociales autónomos, capaces de ejercer una influencia sobre las decisiones políticas, sólo queda la impotencia o la llamada a la indiferencia. ${ }^{4}$

\section{LAS TRES RUPTURAS}

La experiencia contemporánea de la exclusión ha de ponerse en relación con tres auténticos seísmos, que originan circuitos excluyentes como ondas expansivas sobre el resto de la sociedad. ${ }^{5}$ Se trata de un virus mutante que combina en su interior diferentes elementos de muy distinto origen; alguno de carácter estructural o sistémico, otros de tipo relacional o contextual y los terceros que se domicilian en el sujeto, en su capacidad de amar y de esperar, de desear y de soñar. Existen, pues, tres constelaciones de la exclusión: el orillamiento de una organización, la desafiliación de unas relaciones sociales y la desmotivación personal. La exclusión es la radicalización de la pobreza; es la pobreza allí donde ésta se convierte en desgarro personal, en desafiliación co-

3 HERNÁNDEZ ARISTU, LÓPEZ, A.: Formación profesional dual: una intervención reflexiva, Nau Llibres. Valencia, 1998, p. 107.

4 TOURAINE, A.: Comment sortir du liberalisme? Fayard, París, 1999, p. 8-9.

s GARCÍA ROCA, J.: Contra la exclusión. Responsabilidad pública e iniciativa social. Santander, 1995. 
lectiva, en ruptura cultural.

\subsection{Organización excluyente y las fracturas sociales}

La condición de excluido está vinculada, en primera instancia, a la existencia de una organización que orilla y expulsa a personas y grupos. La exclusión se ha instalado en el corazón mismo de la sociedad hasta invertir su orientación inclusiva. ${ }^{6}$ Mientras el sueño de la inclusión era que el crecimiento económico y el bienestar social incorporaría cada vez a más sectores sociales como si viajaran en la misma dirección pero con distinta velocidad, la realidad de la exclusión puede compararse al viaje colectivo de un tren en el que los vagones que transportaban a los mas vulnerables fueron desenganchados.

La existencia de la exclusión expresa y se alimenta de los conflictos sociales, que son las grandes fracturas de carácter estructural, instaladas en la organización social. Cabe señalar aquellas fracturas que tienen mayor importancia en la configuración actual de la exclusión; en primer lugar, el conflicto capital-trabajo inicia una fase en que la productividad del capital crece sin trabajo y lo que es bueno para el capital no lo es para el trabajo (cada mañana se anuncian a la vez las ganancias para las empresas y las reducciones del personal), su población problema son los jóvenes que deambulan buscando un lugar en la sociedad, el parado de larga duración o el joven con una media de 38 meses para encontrar el primer empleo, los trabajadores poco cualificados y los jóvenes con poca experiencia profesional ${ }^{7}$. En segundo lugar, el conflicto Norte-Sur agranda la brecha entre países y cuya población problema es la emigración que llega a nuestras casas como una «pesadilla errante», como desesperación por un lado y de cerrazón por otro. Mientras Brahim, uno de los pocos sobrevivientes del naufragio de una patera el 16 de septiembre de 1998, en nombre de todas las pateras del mundo decía «nadie puede poner fronteras a nuestra hambre» ${ }^{8}$. El conflicto varon-mujer como expresión del dominio de la tribu, que tiene su población problema en la mujer violada y violentada,

6 Foucauld J.B. y PIVETEAU, D.: Une société en quête de sens, Odile Jacob, 1995, p. 144-145.

7 En España el $40 \%$ de las jóvenes y el 36\% de los jóvenes están desempleados, el $39 \%$ y el $30 \%$ en Italia y el $32 \%$ y el $22 \%$, respectivamente, en Francia.

\& En este fin de milenio, el $15 \%$ de la población mundial posee el $79 \%$ de la riqueza; la pobreza absoluta castiga a 1.300 millones de personas a vivir con menos de un dolar por día (lo que cuesta un pasaje de autobús). La fortuna de los tres hombres más ricos del planeta (Bill Gates, el sultán de Brunei y Waren E. Buffet) supera el Producto Interior Bruto (PIB) de los 48 países más pobres, o lo que es lo mismo, que sólo 225 personas acumulan los mismos bienes que 2.6000 millones de sus semejantes. 
en la prostitución forzada, así como la explotación laboral de las mujeres en condiciones de verdadera esclavitud. ${ }^{9}$ Finalmente se anuncia el conflicto entre capital financiero y producción que tiene su población problema en los pueblos declarados improductivos, en especial aquellos territorios como Africa subsahariana que no existen para la globalización económica, y los grupos «económicamente inútiles».

\subsection{Contextos inhabilitantes y la desvinculación}

¿Qué tienen en común las exclusiones actuales? Las exclusiones actuales, de cualquier índole, afectan a las relaciones, al modo de ser y estar en el mundo. Tienen sus vinculaciones sociales rotas y fragilizados los nexos relaciones. La exclusión alude, de este modo, a la presencia masiva y desafiante de la desafiliación, a la ruptura de los contextos y de las relaciones. Especial importancia tiene la fragilización de las redes sociales en todas sus modalidades: redes familiares, vecinales, amistosas que protegían al individuo y le concedían protección, seguridad y libertad. La existencia de unos contextos disgregados, fragmentados, atomizados inhabilitan para el ejercicio de las solidaridades de proximidad. Hay personas que se han visto descolgadas de sus redes naturales como mecanismos de protección general, y se convierten cada vez más en individuos sin apoyos. Es el grado máximo de desafiliación familiar y de ruptura de sus vínculos naturales y de sus redes sociales.

Nos encontramos ante redes que no dan protección ${ }^{10}$ y cuya población problema son las jóvenes madres solteras, que tienen que asumir responsabilidades como madre, con sus necesidades de adolescentes y de cónyuge abandonado, que une al abandono el coraje de sacar sus hijos adelante. Redes que no dan seguridad, cuya población problema son aquellos barrios o zonas de vulnerabilidad, caracterizados por la desafiliación y la ruptura de los vínculos sociales. Redes que no dan libertad sino desanclaje de personas que deambulan como supernumerarios, sin territorio y sin pertenencia, o el nuevo fenómeno de los niños y niñas forzosamente desaparecidos.

\subsection{Subjetividad frágil e insignificancia personal}

¿Qué tienen en común todos los procesos de exclusión? Lo más

9 La participación de las inujeres en las instancias de toma de decisiones no rebasa el $4 \%$, de cada 100 analfabetos en el planeta 66 son mujeres.

1i) El múmero de hogares en España donde todos los miembros activos se encuentran en paro ascendía a finales de 1996 a 973.060 , lo que equivale a un 11\% de todas las familias activas. El numero de familias desprotegidas que no cuenta con ningún preceptor de prestaciones por desempleo es 472.000 , equivalente al $5 \%$ del total de familias. 
visible de la exclusión es que causa la muerte, resulta mortal. Los excluidos de hoy, como los pobres de ayer y los indigentes de anteayer, son los que mueren antes de tiempo (casi 200 millones de personas no llegarán a vivir 60 años), los que dejaron de esperar (vivir es una pesada carga). La exclusión en esta tercera dimensión se hermana con la destrucción de los dinamismos vitales que en última instancia significa muerte en sus distintas manifestaciones: la muerte física, la muerte psíquica, la muerte legal y la muerte social. Muerte física, cuya población problema son los jóvenes que murieron prematuramente y a destiempo, en ciertos lugares no hay tiempo para elaborar el luto" ; la muerte psíquica, cuya población problema son los afectados por las patologías de la subjetividad: la falta de confianza en sí mismo, la inseguridad e incapacidad de lucha, la crisis de identidad personal. Muerte legal, cuya población problema son aquellos colectivos que no han conquistado todavía su reconocimiento legal: los inmigrantes y los adolescentes con problemas con la justicia. Muerte social, que equivale a insignificancia, a no contar socialmente; ser excluido significa no contar para nada, no ser considerado útil a la sociedad, ser descartado de la participación y, sobre todo, sentirse insignificante.

\subsection{Conclusiones}

¿Qué consecuencias prácticas tiene el reconocimiento de esta triple dimensión de la exclusión? A la luz de las explicaciones anteriores, abordar la exclusión de los/as jóvenes significa atender a un tiempo propio, a un estatuto original y a unos procesos concretos.

La lucha contra la exclusión vive hoy el dilema de los tres relojes que Dahrendorf identificó en la reconstrucción de la sociedad possovietica: seis meses pueden ser suficientes para elaborar una reforma constitucional, seis años pueden no bastar para actuar una reforma económica, mientras que la formación y difusión de valores, comportamientos y estilos de vida de una sociedad civil moderna pueden necesitar generaciones enteras. ${ }^{12}$ La lucha contra la exclusión sólo tendrá éxito si acertamos con los tres relojes, si ninguno de ellos se atrasa y ninguna hora se menosprecia: el tiempo largo para revertir la organización a través de la lucha política y económica, el tiempo intermedio para crear nuevas redes sociales o amortiguar la ruptura del tejido social y el tiempo corto para las estrategias de acompañamiento que re-

1) No hay motivos para morir hoy por haber utilizado una jeringuilla de otra persona, pero miles y miles de jóvenes siguen muriendo; no hay motivo para que mi barrio tenga una media de vida de 40 años.

12 DAHRENDORF, R.: Reflections on the revolution in Europe. Clatto and Windus, London 1990. 
hagan las identidades.

En segundo lugar, si ła exclusión nace y se reproduce en el interior de auténticas tramas estructurales, que no sólo crean la exclusión sino que agarrotan a los excluidos, remiten fuera del excluido la razón de su exclusión. Entenderla es perseguir sus tramas, su maraña, enfrentarse a la distribución del poder económico, político y social. No hay solución si no se mueve también la sociedad. Hay una mala representación de las estrategias contra la exclusión que las sitúan en el excluido. Invertimos en sus habilidades, en sus capacidades pero sin mover nada. En la lucha contra la exclusión no sólo se deben mover los excluidos sino todos, incluidos los sistemas educativos y las empresas.

Luchar contra la exclusión es entrar en el mundo de una realidad conflictiva y conflictuada. No se puede trabajar en la exclusión sin provocar conflictos. Sin una voluntad de soportar conflictos, perder amistades, despertar sospechas municipales, perder dinero, el trabajo por la marginación es fácilmente cooptado. Sólo los que puedan estar en el conflicto sin romperse, están llamados a esta apuesta. En la actualidad posmoderna y neoliberal pareciera que mencionar conflictos casi se ha convertido en cosa de mal gusto y poco estético.

\section{APELACIONES AL TERCER SECTOR}

La inserción debe afrontar una organización que orilla y expulsa, unos contextos desagregados e inhabilitantes y unos sujetos desmotivados y apáticos. Si la exclusión se instala en la estructura misma de lo social, desgarra los contextos de proximidad y golpea los dinamismos vitales debe atemperarse cualquier discurso que lo afronte de manera parcial; más bien se necesita golpear a la vez y simultáneamente en todos los frentes de la exclusión con estrategias transversales y movilizadoras de diferentes actores, recursos públicos y privados y administraciones centrales y locales. Sólo la integralidad permite albergar alguna esperanza en la lucha contra la exclusión, ya que las medidas parciales, fragmentadas e incoherentes la retroalimentan y la refuerzan. Actores políticos, económicos, sociales y culturales han de insertarse en una solución integrada; nadie por sí mismo puede ganar la batalla y todos por el contrario son necesarios.

¿Qué papel pueden desempeñar los nuevos actores sociales que se identifican como tercer sector? ¿Cuál es su aportación?

\subsection{El Tercer sector en el escenario del Estado}

Se necesitan medidas de índole política para remover una organiza- 
ción social, que se sostiene sobre una determinada distribución del poder político, económico y cultural. No resulta indiferente al tercer sector la orientación de las políticas generales; cualquier regresión en el nivel de protección aumenta el darwinismo contra los excluidos; cuando en mi barrio se ha cerrado un taller o se ha reducido un subsidio, ha aumentado el desgarro de los excluidos y nunca he podido confirmar que fuera bueno para ellos como proclaman los que les atribuyen el poder de pasivizarles o desmotivarles.

EI Tercer sector tiene en el escenario del Estado una agenda llena y bien repleta. No puede esconder su vocación política, en la medida que tiene voluntad de transformar las organizaciones excluyentes y desarrollar sus competencias en el ámbito político.

\subsubsection{Interlocutor de las políticas públicas}

Si el problema de la exclusión no está en los excluidos, apelar al Tercer sector significa activar tanto la capacidad de presión como de negociación, convertirse en interlocutor de las políticas públicas. En la esfera pública, el Tercer sector se propone representar los intereses de los excluidos, con ellos y desde ellos; en la actualidad, no parece que la población excluida encuentre sus canales de representación a través de los sindicatos, ya que están fuera del mundo laboral, ni tampoco tienen garantizado el acceso a los beneficios del Bienestar, ya que tienen rotos los canales de la información, que les dejan en la antesala del estado de bienestar.

Para ser interlocutor le falta articulación, coordinación y capacidad de crear lo común y le sobra desconfianza hacía el poder político. Para representar al mundo de los excluidos, el tercer sector tiene que convertirse en "partner" de las políticas públicas, con capacidad de crear sinergia con sindicatos, empresas, grupos comunitarios.

La urgencia mayor del Tercer sector ante la realidad de la exclusión consiste en convertirla en una cuestión de interés general, contra la opinión liberal que la reduce a un asunto privado del que la sufre. Construir una sociedad sin marginalidades es un bien común que debe socializarse. Lo cual tiene consecuencias importantes; el Tercer sector es un servicio público que rompeta separación entre lo estatal y lo privado; es el espacio público en el interior de la sociedad civil, una expresión de lo público, que se diferencia de lo estatal y de lo privado; interesa acentuar este aspecto en un momento en el que el Tercer sector se está potenciando como un capítulo de las privatizaciones, cuando realmente es una resistencia a las políticas privatizadoras. En consecuencia, pueden considerarse como un modo de redistribución de los presupuestos públicos, en lugar de alinearse con los que pretenden 
reducirlos. "Sus actividades deben organizarse como medio de redistribución, bajo la égida del estado o de los municipios". ${ }^{13}$

Asimismo, el Tercer sector amplía el ámbito tradicional de lo político, que se identificaba con los partidos y la gestión del poder; la transformación no sólo está servida por la política como gestión del poder sino también por lo pre-político que a veces lo disuelve y lo metapolítico que con frecuencia lo relativiza. Y, sobre todo, trae una nueva representación de las organizaciones públicas más cercana a los ciudadanos y con mayor capacidad de aprendizaje. Cuando los ciudadanos se "apoderen" del Estado, éste será vivido por los ciudadanos como un asunto propio. Por esta razón, el Tercer sector se considera un actor, que profundiza la democracia y la participación ciudadana, complementando a los sindicatos, a los partidos, a los movimientos sociales más tradicionales.

\subsubsection{Un nuevo contrato social}

En su último libro, Ulrich Beck se pregunta “¿Cuánta pobreza podrá soportar la democracia?" ¿Cuánta exclusión, nos preguntamos nosotros, podrá soportar una sociedad cohesionada? Beck sugiere la necesidad de un pacto social contra la exclusión ${ }^{14}$ que contemple algo parecido a un sistema de previsión social de carácter transnacional que incorpore garantías básicas, redes sociales de autoprevision y autoorganización, y un compromiso mayor con la justicia social y económica a escala mundial. No cabe duda que estos asuntos resultarán decisivos para la suerte de los excluidos: la garantía de las rentas mínimas, el reparto del trabajo, la distinción entre trabajo y protección, la consolidación de los sistemas de protección.

La tarea principal del Tercer sector consiste en promover un nuevo contrato social, que, además de estas medidas, reconozca el derecho de inserción como una deuda social, que ocupa una posición intermedia entre el derecho (es accesible a todos) y el contrato (vinculado a una contrapartida a través de su compromiso personal). No es un mero derecho como exigencia ni una ayuda social paternalista, sino que se abre a la implicación recíproca del individuo y de la sociedad a través de estrategias contractuales.

El tercer sector permite inaugurar una nueva concepción del derecho: no son créditos que se pueden exigir sino derechos-proyectos que requieren la implicación de todos los que intervienen. El estado actual de la exclusión requiere la emergencia de nuevos actores que conside- 
ren a los individuos como miembros de una sociedad en la cual han de tener un puesto. El contrato de inserción vincula al excluido a su participación en un proyecto. La lucha contra la exclusión invita a explorar un tercer tipo de derechos que articulan ayuda económica, participación social e implicación personal. Este compromiso personal va desde la formación hasta la participación en actividades de interés general, desde los esfuerzos personales de rehabilitación hasta la promoción de organizaciones sociales. Es un derecho bastante paradójico puesto que está basado en una especie de derecho individualizado y a la vez condicionado. ${ }^{15} \mathrm{La}$ condicionalidad, cuando se trata de ofrecer oportunidades de inserción, se practica con frecuencia como una penalidad y un castigo -se te ofrece una ayuda económica a condición de que cumplas unos deberes o te empeñes en unos compromisos-cuando realmente es la emergencia de un nuevo derecho.

\subsubsection{Políticas culturales}

A los actores sociales corresponde revertir las actuales puertas de entrada a una sociedad excluyente: la seguridad económica como salario de por vida, la participación como consumo de mercancías, la protección como competencia exclusiva del Estado y la identidad personal como individualismo posesivo y competitivo. Se precisan actores culturales, que sean capaces de desarmar a los integrados, de proponerles alternativas contagiosas a sus modos de vivir y de acaparar; al final la historia será la batalla sobre la felicidad: quién ofrece más felicidad, quién enseña a competir y en consecuencia a excluir, o quién vive solidariamente. Es una cuestión que afecta a la persona y cuando se trata de "transformación de personas no se puede controlar causal y técnicamente por medio del derecho y el dinero". ${ }^{16}$

El Tercer sector es también una realidad cultural, capaz de remover los factores de inclusión: el trabajo, como única entrada a la renta económica, a la identidad social, a las expectativas sociales, el consumo como marca de prestigio y posición social que consiente que 250 personas dispongan de lo mismo que la mitad de la población mundial, la protección que se delega al Estado con olvido de las instituciones intermedias, y la identidad personal construida desde la autoafirmación excluyente en lugar de la cooperación.

15. ROSANVALLON: La revolución del derecho a la inserción, en Debats, 54 (1996), p.40.

16 LUHMANN, N.: Teoria politica en el Estado de Bienestar. Alianza Universidad, Madrid, 1993 , p.105. 


\subsection{El Tercer sector en el escenario económico}

Al suscitar, sostener y animar iniciativas socio-económicas, el Tercer sector se convierte en un ámbito necesario para las estrategias de inserción. Se trata de colocar la utilidad social en el centro de lo económico. Lo cual tiene consecuencias importantes sobre ciertos jóvenes que están condenados a la inactividad y a deambular desorientados por las periferias del bienestar.

La actividad económica del Tercer sector contribuye decididamente a la integración social en la medida que en el origen de la exclusión está el desempleo que obstruye la puerta de entrada a los beneficios sociales (renta económica, posición social, protección...), el aislamiento que rompe los vínculos sociales y la crisis de sentido con la consiguiente inadaptación y anomia.

¿Qué papel desempeña la economía social para las medidas de inserción? ¿De qué forma contribuye a combatir la desocupación, romper el aislamiento y combatir la inadaptación?

\subsubsection{Nuevas formas laborales}

La relación con el trabajo constituye un elemento sustantivo en el origen de la exclusión, ya que en muchos casos empieza siendo un problema laboral y en la medida que el trabajo es la puerta de entrada a la organización social, se convierte en un problema de insignificancia social y crisis de identidad personal.

Si el trabajo se identifica únicamente con su forma salarial se estrecha la puerta de entrada a la integración y se dificulta la situación de los desempleados cuando se identifica estar integrado con ser asalariado. El surgimiento de la sociedad salarial ha impulsado una seria reducción de las modalidades del trabajo, de forma que tener trabajo ha acabado significando tener un salario, un contrato de por vida, vacaciones inteligentes y una organización de la que depende su seguridad. ${ }^{17}$

La aportación más significativa del Tercer sector a las medidas de inserción consiste en ampliar las formas de trabajo y las modalidades de la ocupación más allá de la forma salarial; el tercer sector reintroduce la problemática de las formas de trabajo y amplia el concepto mismo de ocupación a través de otras formas como el trabajo colectivo, la actividad ocupacional, los servicios de autoayuda, la producción no monetarizada... El trabajo no se crea sólo mediante la salarización y la

17 CASTEL, R.: Les métamorphoses de la question sociale. Une chronique du salariat. Fayard, París, 1995 
mercantilización, este prejuicio será una fuente constante de marginalidad.

La economía de mercado y la sociedad salarial no bastan para asegurar la ocupación, conviene complementarla con otras actividades que obedecen a otras reglas para satisfacer necesidades colectivas. El último informe al Club de Roma sobre el dilema del empleo trata de superar este equívoco que ha tenido graves consecuencias para las políticas de integración. La mentalidad de la revolución industrial ha tenido graves consecuencias al no valorar en términos económicos las aportaciones no monetarizadas ni las contribuciones no remuneradas. Hoy asistimos a la necesidad de revalorizar las actividades productivas no remuneradas por distintas razones; en primer lugar, por razones estrictamente económicas, ya que el valor económico no puede ligarse solamente al trabajo y a las actividades productivas medidas con un criterio monetario, sino también a los servicios que son intrínsecos al sistema de producción: el estado de salud, la autoestima, la confianza... En segundo lugar, por razones sociales "sólo cuando empecemos a valorar por igual las aportaciones no remuneradas y las remuneradas podemos reconocer plenamente la participación del trabajo de la mujer y su papel en nuestra economía". Y en tercer lugar por razones políticas; los elevados costes de los servicios pro-bienestar diseñados exclusivamente desde los gastos institucionales hacen cada vez menos viable el sistema de bienestar. Se necesita incorporar el sistema no monetarizado para salvarnos de la ineficacia del sistema monetarizado; de este modo resulta productivo la incorporación de la atención domiciliaria a los tratamientos hospitalarios, o la movilización de los abuelos y las abuelas para realizar los cuidados de los pequeños. ${ }^{18}$

\subsubsection{Nuevas vinculaciones sociales}

El Tercer sector se propone inscribir la solidaridad en el corazón de la economía; la actividad económica puede y debe ejercerse en función de finalidades sociales y mejora de las condiciones de vida. Hay grupos que se organizan como economía solidaria porque entienden que el mundo económico no puede abandonarse a las leyes de las ganancias y del beneficio privado, ni tampoco estamos condenados a corregir los efectos perversos mediante los mecanismos de protección activados por el Estado de bienestar sino que intentan incidir en la lógica misma de lo económico. Ni las empresas tiene el monopolio en la creación de empleo ni el Estado tiene el monopolio del interés público. ${ }^{19}$

1s GIARINI, LIEDTKE: El dilema del empleo. El fituro del trabajo. Galaxia Gutem. Barcelona, 1998, pp. 134 y 161.

19) LAVILLE, J.L. L'Économie solidaire. Desclée de Brouwer, París, 1998, p.141. 
La economía ha sido uno de los elementos básicos en la creación del individualismo posesivo en cuanto que ha consagrado la competitividad y el beneficio individual. La economía solidaria se basa sobre la confianza personal en lugar de los intereses, sobre la colaboración en lugar de la competencia. Sin renunciar a la lógica económica, combina eficiencia y contribución a la integración, profesionales y voluntarios, principios económicos y principios sociales de cuyas sinergias mutuas recibe su acreditación.

En lugar de una economía exclusivamente selectiva que refuerza la diferencia entre privilegiados y excluidos, el tercer sector apuesta por una economía social que, a pequeña escala, inaugure otra lógica del intercambio.

\subsubsection{Integración autoregulada}

En la exclusión quedan afectados los dinamismos vitales que frecuentemente se traducen en procesos de inadaptación social y en síntomas de desestructuración personal. Aunque no es bueno ni razonable identificar las actividades económicas de utilidad social con la intervención en las personas excluidas, es evidente que resulta un laboratorio para la reconstrucción de la identidad personal y de la autoestima.

Frente a la esfera heterónoma, que representa el mercado tradicional y la esfera económica tradicional, el tercer sector ensaya la autoimplicación como una forma apropiada para dinamizar los procesos de inserción. Los excluidos pasan de objetos de atención a sujetos y protagonistas de su propio destino; pasan de socios a ciudadanos activos. De este modo se afianza su autoestima y se recrean sus dinamismos vitales, siempre que se puedan establecer otros fines y criterios no medibles únicamente en términos económicos ni a través de la lógica monetaria. Pasar de la heteronomía a la autoregulación está en el centro de todo proceso insertivo.

La ventaja comparativa del Tercer sector está en combinar el trabajo profesional con la intervención familiar, hermanar asalariados con voluntarios, pero sobre todo poner en manos de los excluidos la decisión acerca de sus propios destinos.

\subsection{El Tercer sector en el escenario de los mundos vitales}

Los proyectos de inserción incorporan un momento, que afecta a la resocialización, a la reconstrucción de la identidad y a los dinamismos vitales. ¿Qué puede aportar el Tercer sector a la reconstrucción de la identidad personal y relacional? 


\subsubsection{Creación de vínculos sociales}

Las estrategias más acreditadas en el campo de la exclusión son las prácticas reticulares, que se enfrentan a la desafiliación y a la ruptura de las redes sociales. La lucha contra la exclusión se impone como tarea activar entornos, reconstruir el medio ambiente interhumano, recrear las redes de dependencia afectivas y el entorno comunicativo.

Para reconstruir las redes sociales, el Tercer sector aporta la gestión de la inclusión desde lo local, desde las potencialidades locales y desde el desarrollo de actividades autogestionadas; sólo la movilización de los recursos locales para tratar «in situ» un problema de exclusión, resulta pertinente. Y, en segundo lugar, sugiere la reconstrucción de los enclaves afectivos y simbólicos para la resocialización y recreación de las motivaciones; para ello son esenciales los recursos educativos y culturales, y la creación de espacios de sociabilidad que hagan soportable la cotidianeidad, ayuden a abrirse a la singularidad y refuercen la autoestima, ya que no siempre el tipo de trabajo contribuye a la autoestima. ${ }^{20}$

Las organizaciones del Tercer sector son instituciones de mediación que facilitan también las pasarelas para activar los factores de inserción, y superar el aislamiento social y la guetización de los excluidos.

\subsubsection{Acompañamiento}

El territorio del asociacionismo es un espacio inmantado por el encuentro de personas y la comunicación entre ellas. Los procesos de inserción que potencian el Tercer sector en los mundos vitales se sostienen sobre la reconstrucción de los valores desde la proximidad en el interior de los problemas cotidianos: el excluido, como cualquier ser vivo, necesita de oxígeno, agua, alimento; pero como ser humano necesita de reconocimiento, que es un derecho y un deber de la vida cotidiana. Y, finalmente, alude al uso delicado de la fuerza en el interior del conflicto social; no sitúa más allá del conflicto, ya que éste es parte de la realidad, sino que obliga a estar atentos a que no rompa al excluido. Los excluidos piden instituciones «tiernas» frente a instituciones desgarradoras, frías, distantes.

En los procesos de inserción es necesario activar una ecología del espíritu, que promueva procesos de identidad personal y colectiva en el interior de la trama de significaciones. Sobre todo los jóvenes precisan de esferas personales interesadas por la afirmación de la identidad 
personal en el interior de una comunidad humana. Necesitan una identidad abierta, que se deja asaltar por el grito de los últimos y de los que están peor situados, aunque sea una identidad fracturada por la diferencia y tensionada por la desigualdad.

\subsubsection{Relación de ayuda}

El Tercer sector no puede ser el remedio a los desgarros de la exclusión, en sus manos sólo está promover medidas y acompañar personas que permita hacerse cargo de sí mismo, apoderarse de sus propias competencias.

No tiene el poder de construir una alternativa al mercado total, pero tiene la fuerza para rechazar su lógica y racionalidad cuando impregna a toda la sociedad. En el campo de la exclusión, la solidaridad es más promoción de proyectos que oposición política.

Un gran número de jóvenes se encuentran arrastrados por relaciones que no son capaces de entender, dominar o ignorar por sus propios medios o capacidades. Los excluidos no están en muchos casos en condiciones para ayudarse a sí mismos, necesitan ser ayudados. ¿Qué relación de ayuda resulta necesaria y positiva? Las personas y grupos excluidos han creado un nuevo concepto de ayuda que es inseparable de la participación, función tutorial y relaciones afectivas.

La ayuda acreditada es inseparable de la participación. Las prácticas de inserción no son ni un subsidio asistencial ni una prestación de Seguridad Social. Hay un compromiso recíproco del individuo y de la colectividad, teniendo en cuenta las necesidades, aspiraciones y posibilidades de los beneficiarios. Capacita a los excluidos para su propia autoorientación y autoregulación, y crea en ellos la capacidad y la actitud activa hacia la toma de decisiones sobre su propio futuro.

La relación de ayuda en el ámbito de la exclusión es inseparable de la función tutorial, que entraña una relación individualizada con la persona excluida, con sus actitudes, aptitudes, conocimientos e intereses. La acción tutorial debe atender a las peculiares características de cada persona excluida. Implica de manera coordinada a las distintas personas e instituciones educativas, sanitarias, culturales y laborales. Cuando se tienen en cuenta las necesidades, aspiraciones y posibilidades del individuo, se inaugura una nueva relación de ayuda individualizada, casi tutorial. Ya no es posible una gestión homogénea, burocrática ni tecnocrática que nos enmarca a los excluidos en categorías juridicoadministrativas. Se trata de un itinerario adaptado a las necesidades de la persona que depende de la situación de cada uno. Es un itinerario individualizado ya que no existe la exclusión sino los excluidos. Lo cual exige un cambio de residencia mental que afecta no sólo a la orien- 
tación general de las políticas sociales sino a la propia intervención y al estilo mismo de la acción social. El compromiso del Tercer sector consiste en entrar en el mundo de la exclusión por la puerta de la amistad; lo cual trae consigo algo importante, la amistad. No hay auténtica relación de ayuda en el ámbito de la exclusión si no está unida a la amistad. Sólo la amistad introduce en la constelación de la exclusión que no sólo invoca los mecanismos económicos y abstractos sino la celebración de la dignidad que requiere actividades basadas en el don y en la reciprocidad. ${ }^{21}$ 